\title{
Dihydroartemisinin and gefitinib synergistically inhibit NSCLC cell growth and promote apoptosis via the Akt/mTOR/STAT3 pathway
}

\author{
HONG JIN $^{1}$, AI-YING JIANG ${ }^{2}$, HAN WANG $^{3}$, YONG CAO ${ }^{4}$, YAN WU $^{5}$ and XIAO-FENG JIANG ${ }^{1}$ \\ ${ }^{1}$ Department of Clinical Laboratory, The Fourth Affiliated Hospital of Harbin Medical University, Harbin, \\ Heilongjiang 150001; ${ }^{2}$ Department of Pneumology, Hongqi Hospital; Departments of ${ }^{3}$ Clinical Skills Center, \\ ${ }^{4}$ Pathology and ${ }^{5}$ Medical Research Center, Mudanjiang Medical University, Mudanjiang, Heilongjiang 157011, P.R. China
}

Received March 9, 2016; Accepted February 20, 2017

DOI: $10.3892 / \mathrm{mmr} .2017 .6989$

\begin{abstract}
Non-small cell lung cancer (NSCLC) is among the leading causes of cancer-associated mortality worldwide. In clinical practice, therapeutic strategies based on drug combinations are often used for the treatment of various types of cancer. The present study aimed to investigate the effects of the combination of dihydroartemisinin (DHA) and gefitinib on NSCLC. Cell Counting kit 8 assay was used to evaluate cell viability. Transwell assays were performed to investigate cellular migration and invasion, and cellular apoptosis was evaluated using the terminal deoxynucleotidyl transferase dUTP nick-end labeling assay. Flow cytometry was used to investigate cell cycle distribution and the expression levels of target proteins were determined using western blot analysis. The results of the present study demonstrated that DHA (5, $10,20,50$ and $100 \mu \mathrm{M})$ reduced cancer cell viability in a dose-dependent manner in the NCI-H1975 human NSCLC cell line and significantly enhanced gefitinib-induced apoptosis. Furthermore, DHA and gefitinib co-administration induced cell cycle arrest in $\mathrm{G}_{2} / \mathrm{M}$ phase, which was associated with a marked decline in the protein expression levels of $\mathrm{G}_{2} / \mathrm{M}$ regulatory proteins, including cyclin $\mathrm{B} 1$ and cyclin-dependent kinase 1 . The addition of DHA appeared to potentiate the inhibitory actions of gefitinib on the migratory and invasive capabilities of NCI-H1975 cells. DHA and gefitinib co-administration also downregulated the expression levels of phosphorylated (p)-Akt, p-mechanistic target of rapamycin, p-signal transducer and activator of transcription 3 and $\mathrm{B}$-cell lymphoma 2 (Bcl-2), and upregulated the
\end{abstract}

Correspondence to: Dr Xiao-Feng Jiang, Department of Clinical Laboratory, The Fourth Affiliated Hospital of Harbin Medical University, 37 Yiyuan Street, Harbin, Heilongjiang 150001, P.R. China

E-mail: lulu98123456@163.com

Key words: non-small cell lung cancer, dihydroartemisinin, gefitinib, Akt, mTOR, STAT3 expression of Bcl-2-associated $\mathrm{X}$ protein. In conclusion, the present results suggested that the combination of DHA and gefitinib may have potential as a novel and more effective therapeutic strategy for the treatment of patients with NSCLC.

\section{Introduction}

According to GLOBOCAN, lung cancer is among the most common types of cancer worldwide (1). Non-small cell lung cancer (NSCLC) comprises $85 \%$ of all types of lung cancer. NSCLC is relatively insensitive to chemotherapy compared with small cell lung cancer $(2,3)$. Despite recent advances in the chemotherapy of NSCLC, the therapeutic efficacy of currently available agents remains unsatisfactory and the majority of patients with advanced NSCLC are refractory to medication $(4,5)$. Therefore, the development of novel therapeutic strategies characterized by higher efficacy is imperative for the treatment of patients with NSCLC.

Gefitinib (ZD1839, Iressa) is a chemotherapeutic agent that was approved by the US Food and Drug Administration in 2003 for the treatment of patients with local or metastatic NSCLC, following failure of treatment with platinum- and docetaxel-based chemotherapeutic schemes (6). Gefitinib is an effective and well-tolerated agent; however, some patients with NSCLC are insensitive to gefitinib chemotherapy (7). Dihydroartemisinin (DHA) is the active metabolite of all artemisinin compounds and is widely used as an antimalarial therapeutic agent. In addition, previous studies have reported that DHA exhibited pronounced anticancer effects in breast, colorectal, cervical and lung cancer (8-11), whereas in combination with chemotherapeutic agents it exerted synergistic effects in the treatment of various types of cancer $(12,13)$.

The NCI-H1975 human NSCLC cell line was established in July 1988, and is characterized by much lower sensitivity to gefitinib than other human NSCLC lines, such as A431 and H3255 (14). The present study aimed to ascertain whether the combination of gefitinib with DHA have a higher therapeutic efficacy than currently used gefitinib monotherapy. Therefore, the present study investigated the combination of gefitinib with DHA to improve the chemotherapeutic sensitivity of 
NCI-H1975 cell, and explored the molecular mechanisms underlying actions of the drugs.

\section{Materials and methods}

Cell culture. The NCI-H1975 human lung adenocarcinoma cell line was purchased from American Type Culture Collection (Manassas, VA, USA) and cultured in RPMI-1640 medium (catalog no. SH30809.01; HyClone; GE Healthcare Life Sciences, Logan, UT, USA), supplemented with $10 \%$ fetal bovine serum (FBS; HyClone; GE Healthcare Life Sciences). NCI-H1975 cells were maintained at $37^{\circ} \mathrm{C}$ in a humidified $5 \%$ $\mathrm{CO}_{2}$ atmosphere.

Cell Counting kit 8 (CCK8) assay. Cellular viability was evaluated using CCK8 (Beyotime Institute of Biotechnology, Haimen, China), according to the manufacturer's protocol. Briefly, NCI-H1975 cells were seeded at $5 \times 10^{3}$ into 96-well plates containing RPMI 1640 medium, supplemented with $10 \%$ FBS and incubated for $24 \mathrm{~h}$. When NCI-H1975 cells reached $80 \%$ confluence they were treated with gefitinib (catalog no. HY-50895; ApexBio; MedChemExpress, New Jersey, USA) or DHA (catalog no.S2290; Selleck Chemicals, Houston, TX, USA) for $24 \mathrm{~h}$. Subsequently, viable cells were detected using CCK8. The absorbance of each sample at $450 \mathrm{~nm}$ was measured using a microplate reader (Tecan Group Ltd., Salzburg, Austria).

Terminal deoxynucleotidyl transferase dUTP nick-end labeling (TUNEL) assay. NCI-H1975 cells were incubated with DHA and gefitinib for $24 \mathrm{~h}$ to induce cell apoptosis. Cellular apoptosis was assessed via evaluating DNA fragmentation in NCI-H1975 cells, using a Cell Death Detection kit (catalog no. 11684817910; Roche Diagnostics, Basel, Switzerland), as previously described (15). Briefly, cells were fixed for $24 \mathrm{~h}$ with $4 \%$ paraformaldehyde at room temperature, washed with PBS and permeabilized for 30 min with $1 \%$ Triton $\mathrm{X}-100$ at $4^{\circ} \mathrm{C}$. Subsequently, cells were treated with the terminal deoxynucleotidyl transferase-labeled nucleotide mix and maintained at $37^{\circ} \mathrm{C}$ for $1 \mathrm{~h}$ in the dark. Slides were rinsed and counterstained (TE 2000-U; Nikon Corporation, Tokyo, Japan) for $15 \mathrm{~min}$ with $10 \mathrm{mg} / \mathrm{ml}$ 4,6-diamidino-2-phenylindole (DAPI) at $37^{\circ} \mathrm{C}$.

Cell cycle analysis. The effects of gefitinib and DHA on cell cycle distribution were assessed using flow cytometric analysis of the DNA content of NCI-H1975 cells, following staining with propidium iodide (PI). Briefly, NCI-H1975 cells were seeded in 6-well plates and allowed to attach overnight at $37^{\circ} \mathrm{C}$. Fresh complete RPMI-1640 medium was then added, containing the desired concentrations of gefitinib $(10 \mu \mathrm{M})$ or DHA $(10 \mu \mathrm{M})$, and cells were incubated for $24 \mathrm{~h}$ at $37^{\circ} \mathrm{C}$. Cells were then washed with PBS and fixed in $70 \%$ ethanol overnight at $4^{\circ} \mathrm{C}$. Subsequently, cells were treated at room temperature with $80 \mathrm{mg} / \mathrm{ml} \mathrm{RNaseA}$ and $50 \mathrm{mg} / \mathrm{ml}$ PI for $30 \mathrm{~min}$, and analyzed (ModFitLT version 2.0; Verity Software House Inc., Topsham, ME, USA) using a Coulter ${ }^{\circledR}$ Epics $^{\circledR} \mathrm{XL}^{\mathrm{TM}}$ Flow Cytometer (Beckman Coulter, Inc., Brea, CA, USA).

Cell migration and invasion analysis. To assess the effect of chemotherapeutics on the migratory and invasive capabilities of NCI-H1975 cells, cells were incubated with gefitinib $(10 \mu \mathrm{M})$ or DHA $(10 \mu \mathrm{M})$ at $37^{\circ} \mathrm{C}$ for $24 \mathrm{~h}$. NCI-H1975 cells were harvested, resuspended in RPMI-1640 medium and seeded at $5 \times 10^{5}$ into the upper chambers of Transwell inserts with $8 \mu \mathrm{m}$ pore size (EMD Millipore, Billerica, MA, USA). The lower chambers contained culture medium supplemented with $10 \%$ FBS as a chemoattractant. Following incubation at $37^{\circ} \mathrm{C}$ in $5 \%$ $\mathrm{CO}_{2}$ for $24 \mathrm{~h}$, non-migrated cells on the top of the membrane were removed with cotton swabs. Cells that had migrated to the lower membrane were fixed with $95 \%$ ethanol, stained for $1 \mathrm{~h}$ with $0.2 \%$ crystal violet (Sigma-Aldrich; Merck KGaA) and counted under a light microscope. Each experiment was performed in triplicate. Matrigel-coated 24-well Boyden chambers (EMD Millipore) were used in the cell invasion assay. Cells were seeded in the upper chamber in serum-free medium. The lower chambers contained culture medium supplemented with $10 \%$ FBS as a chemoattractant. Following incubation at $37^{\circ} \mathrm{C}$ in $5 \% \mathrm{CO}_{2}$ for $24 \mathrm{~h}$, non-invasion cells on the top of the membrane were removed with cotton swabs. After $24 \mathrm{~h}$, cells that had invaded to the lower membrane were fixed, stained with $0.2 \%$ crystal violet (Sigma-Aldrich; Merck KGaA, Darmstadt, Germany) for $1 \mathrm{~h}$, and counted (Image-Pro Plus 6.0, Media Cybernetics, Inc., Rockville, MD, USA) under a light microscope.

Western blot analysis. Total protein was extracted from NCI-H1975 cells. NCI-H1975 cells were digested with lysis buffer (catalog no. P0013B; Beyotime Institute of Biotechnology). Briefly, the NCI-H1975 cells were harvested and centrifuged at $12,000 \times \mathrm{g}$ at $4^{\circ} \mathrm{C}$ for $15 \mathrm{~min}$ and the supernatant was collected for the western blot experiment. Total protein concentration in the supernatant was determined using a Bicinchoninic Acid assay (BCA; Beyotime Institute of Biotechnology). Briefly, the BCA working solution was prepared at a ratio of 50:1 with BCA reagent $\mathrm{A}$ and $\mathrm{BCA}$ reagent $\mathrm{B}$, respectively, and mixed thoroughly. Following this, $200 \mu 1$ of BCA working solution was added to each of the 96-well plates containing protein samples, which were incubated at $37^{\circ} \mathrm{C}$ for $30 \mathrm{~min}$. The absorbance was measured at a wavelength of $562 \mathrm{~nm}$ by a microplate reader, and the protein concentration of the sample was calculated from the standard curve. A total of 60-80 $\mu \mathrm{g}$ extracted protein samples were separated by $10-15 \%$ SDS-PAGE and transferred onto nitrocellulose membranes. The membranes were blocked with $5 \%$ non-fat milk at room temperature for $1 \mathrm{~h}$. Subsequently, the membranes were incubated at $4^{\circ} \mathrm{C}$ overnight with the following primary antibodies: Anti-mechanistic target of rapamycin (mTOR; 1:1,000; catalog no. 9964; Cell Signaling Technology, Inc., Danvers, MA, USA), anti-phosphorylated (p)-mTOR (1:1,000), anti-cyclin B1, anti-B-cell lymphoma 2 (Bcl-2; 1:1,000; catalog no. 2872; Cell Signaling Technology, Inc.) anti-Bcl-2-associated X protein (Bax; 1:1,000; catalog no. 2774; Cell Signaling Technology, Inc.) anti-signal transducer and activator of transcription (STAT) 3 (1:1,000; catalog no. ab119352; Abcam, Cambridge, MA, USA), anti-p-STAT3 (1:1,000; catalog no. ab30647; Abcam), anti-cyclin-dependent kinase (Cdk) 1 (1:1,000; catalog no. ab18; Abcam) and anti- $\beta$-actin (1:4,000; KangChen Bio-tech, Inc., Shanghai, China). Membranes were washed three times for $10 \mathrm{~min}$ in PBS containing $0.5 \%$ Tween-20, and incubated with Alexa 
A

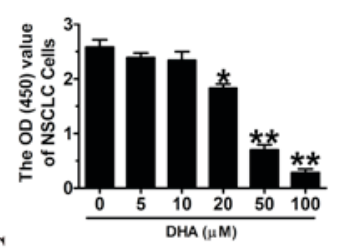

C

B

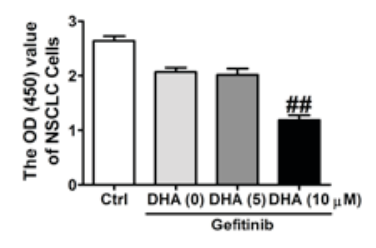

A

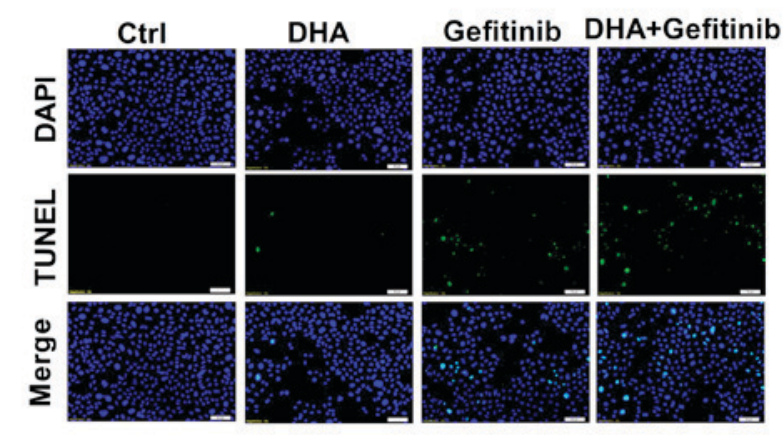

B

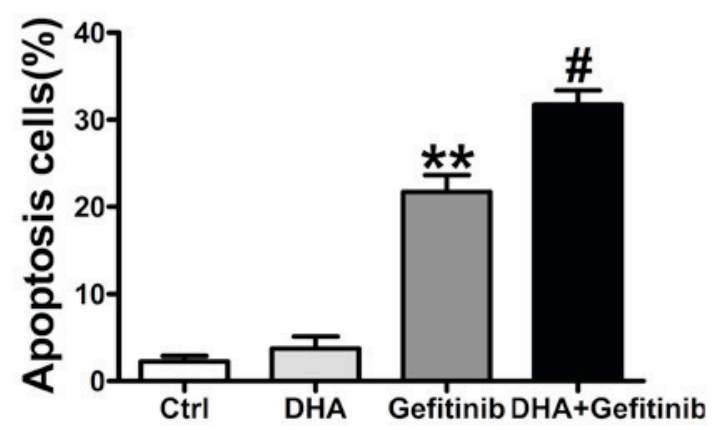

Fluor $^{\mathrm{TM}}$-conjugated secondary antibody (Invitrogen; Thermo Fisher Scientific, Inc., Waltham, MA, USA) for $1 \mathrm{~h}$ at room temperature. IRDye ${ }^{\circledR} 800 \mathrm{CW}$ Goat anti-Rabbit (catalog nos. 926-32211, C60607-15; LI-COR Biosciences, Lincoln, NE, USA). IRDye ${ }^{\circledR} 800 \mathrm{CW}$ Goat anti-Mouse (catalog nos. 926-32210, C60405-05; LI-COR Biosciences). The bands were visualized using the Odyssey Imaging system (LI-COR Biosciences) and semi-quantified using the Odyssey software version 3.0. Relative protein expression was normalized to $\beta$-actin, which was used as an internal control.

Statistical analysis. The statistical significance of the difference between groups was assessed by one-way analysis of variance, followed by Dunnett's test for multiple comparisons. Data are expressed as the mean \pm standard error of the mean. $\mathrm{P}<0.05$ was considered to indicate a statistically significant difference.

\section{Results}

DHA inhibits NCI-H1975 cellular proliferation. To investigate whether DHA may inhibit the growth of lung cancer cells, CCK8 assay was performed. Results demonstrated that DHA significantly inhibited NCI-H1975 cellular viability in a dose-dependent manner (Fig. 1A). The combination of DHA $(10 \mu \mathrm{M})$ and gefitinib $(10 \mu \mathrm{M})$ exhibited significantly enhanced inhibitory effects on NCI-H1975 cells compared with gefitinib alone (Fig. 1B); however, a lower dose of DHA ( $5 \mu \mathrm{M})$, did not appear to potentiate the effects of gefitinib (Fig. 1B).

DHA potentiates the gefitinib-induced apoptosis of NCI-H1975 cells. In accordance with previous studies (16-19), Co-treatment of DHA with gefitinib significantly increased cancer cell apoptosis. Treatment with a combination of DHA $(10 \mu \mathrm{M})$ and gefitinib resulted in significantly higher cancer

Figure 2. DHA enhanced the gefitinib-induced apoptosis of NCI-H1975 cells. (A) Representative photomicrographs of TUNEL-positive NCI-H1975 cells. All scale bars $=50 \mu \mathrm{m}$. (B) Quantitative analysis of apoptotic NCI-H1975 cells. The apoptotic rate was higher among cells treated with the combination of DHA and gefitinib compared with cells treated with DHA or gefitinib alone. Cells in the $\mathrm{Ctrl}$ group received no treatment. Data are expressed as the mean \pm standard error of the mean of 6 independent experiments. ${ }^{* *} \mathrm{P}<0.01$ vs. the Ctrl group; ${ }^{*} \mathrm{P}<0.05$ vs. the gefitinib group. DHA, dihydroartemisinin; TUNEL, terminal deoxynucleotidyl transferase dUTP nick-end labeling; Ctrl, control.

cell apoptotic rate compared with treatment with gefitinib alone. DHA administered alone demonstrated no marked effect on cell apoptosis (Fig. 2B). Representative images were indicated in Fig. 2A. These results suggested that DHA may enhance the proapoptotic effects of gefitinib, and therefore may have potential as an agent for adjuvant therapy in NSCLC.

DHA potentiates the gefitinib-induced downregulation of cyclin B1 and Cdk1 expression in NCI-H1975 cells. Cell cycle progression of NCI-H1975 cells is controlled by the sequential activation of Cdks, whose activity depends on their association with regulatory cyclins. The formation of a Cdk1-cyclin B1 complex is crucial for the initiation of mitosis in several organisms $(20,21)$. The analysis of cell cycle distribution using flow cytometry revealed a $\mathrm{G}_{2} / \mathrm{M}$ arrest in NCI-H1975 cells treated with gefitinib or a combination of gefitinib and DHA (Fig. 3A and Table I). To investigate the underlying mechanisms, Cdk1 and cyclin B1 expression was assessed using western blot analysis (Fig. 3B). Treatment of NCI-H1975 cells with gefitinib for $24 \mathrm{~h}$ resulted in a significant increase in the percentage of cells in $\mathrm{G}_{2} / \mathrm{M}$ phase, accompanied by a decrease in the percentage of cells in $\mathrm{G}_{0} / \mathrm{G}_{1}$ phase. These effects were potentiated following DHA 
Table I. Effects of DHA and gefitinib on cell cycle distribution of NCI-H1975 cells.

Proportion of cells $(\%)$

\begin{tabular}{llcl}
\cline { 2 - 4 } Group & $\mathrm{G}_{0} / \mathrm{G}_{1}$ phase & S phase & $\mathrm{G}_{2} / \mathrm{M}$ phase \\
\hline Ctrl & $75.54 \pm 1.12$ & $15.01 \pm 2.01$ & $10.96 \pm 1.27$ \\
DHA & $73.02 \pm 3.15$ & $15.58 \pm 1.61$ & $11.75 \pm 1.86$ \\
Gefitinib & $67.21 \pm 1.07^{\mathrm{a}}$ & $15.94 \pm 2.46$ & $18.98 \pm 1.42^{\mathrm{a}}$ \\
DHA+Gefitinib & $60.92 \pm 1.27^{\mathrm{b}}$ & $16.33 \pm 1.85$ & $21.73 \pm 1.03^{\mathrm{b}}$ \\
\hline
\end{tabular}

${ }^{\mathrm{a}} \mathrm{P}<0.01$ vs. the Control group; ${ }^{\mathrm{b}} \mathrm{P}<0.05$ vs. the gefitinib group. Data are expressed as the mean \pm standard error of the mean. DHA, dihydroartemisinin.
A
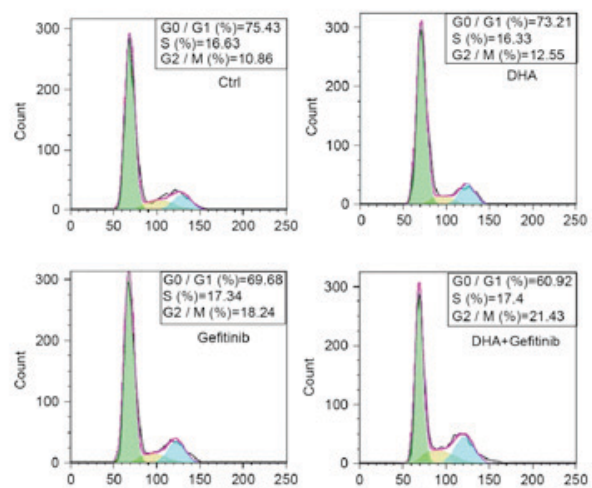

B
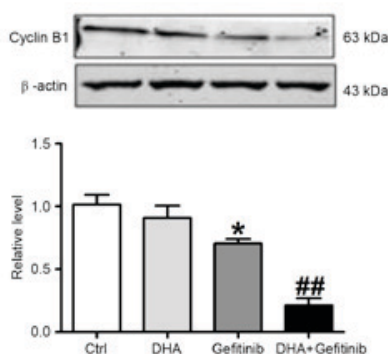

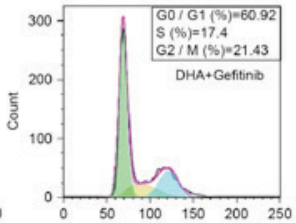

C
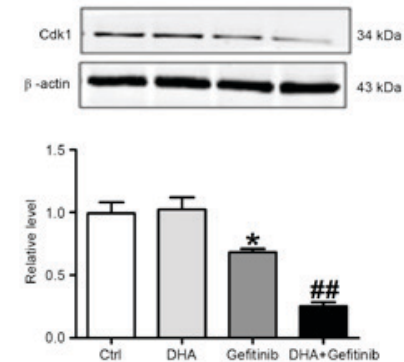

Figure 3. Effects of DHA and gefitinib on NCI-H1975 cell cycle distribution. (A) Representative graphs demonstrate NCI-H1975 cells treated with DHA or gefitinib alone or in combination for $24 \mathrm{~h}$. Cells in the Ctrl group received no treatment. (B) Cyclin $\mathrm{B} 1$ and (C) Cdk1 protein expression levels were significantly downregulated in NCI-H1975 cells treated with the combination of DHA $(5 \mu \mathrm{M})$ and gefitinib $(10 \mu \mathrm{M})$. Data are expressed as the mean \pm standard error of the mean of 6 independent experiments. ${ }^{*} \mathrm{P}<0.05$ vs. the Ctrl group; ${ }^{\# \#} \mathrm{P}<0.01$ vs. the gefitinib group. DHA, dihydroartemisinin; Ctrl, control; Cdk, cyclin-dependent kinase.

and gefitinib co-treatment (Fig. 3A). Furthermore, western blot analysis demonstrated that treatment of NCI-H1975 cells with gefitinib $(10 \mu \mathrm{M})$ caused a significant downregulation in cyclin B1 and Cdk1 protein expression levels. Notably, DHA co-administration significantly potentiated the effects of gefitinib on cyclin B1 and Cdk1 downregulation (Fig. 3B and C). These results suggested that the addition of DHA to gefitinib chemotherapy may potentiate the downregulation of cyclin B1 and $\mathrm{Cdk} 1$ protein levels and prevent Cdk1-cyclin B1 complex formation, and thus promote $\mathrm{G}_{2} / \mathrm{M}$ phase arrest in cancer cells.
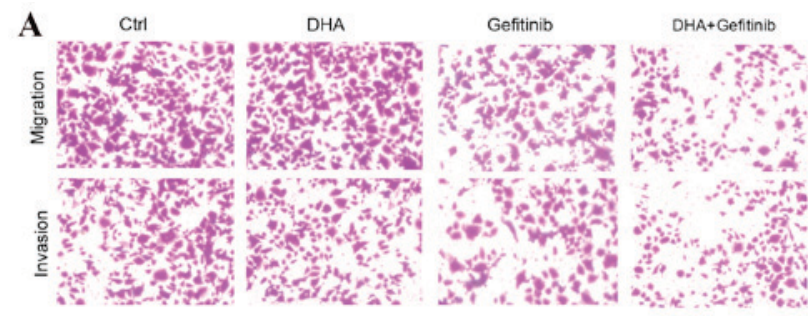

B

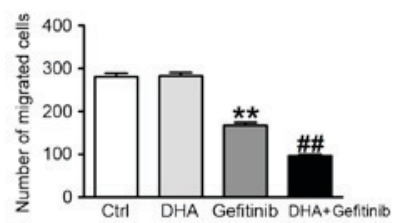

C

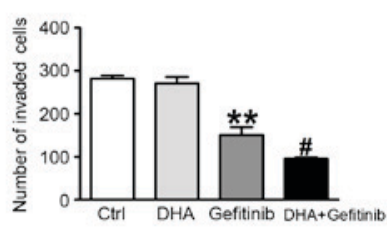

Figure 4. DHA enhanced gefitinib-induced inhibition of NCI-H1975 cell migration and invasion. (A) Representative photomicrographs demonstrating the alterations in cellular migration and invasion following treatment with DHA or gefitinib alone or in combination. Cells in the Ctrl group received no treatment. All scale bars $=50 \mu \mathrm{m}$. (B) Inhibitory effects of gefitinib on cancer cell migration were significantly enhanced following DHA co-administration. (C) Inhibitory effects of gefitinib on cancer cell invasion were significantly enhanced following DHA co-administration. Data are expressed as the mean \pm standard error of the mean of 6 independent experiments. ${ }^{* *} \mathrm{P}<0.01$ vs. the $\mathrm{Ctrl}$ group; ${ }^{\#} \mathrm{P}<0.05,{ }^{\# \#} \mathrm{P}<0.01$ vs. the gefitinib group DHA, dihydroartemisinin; Ctrl, control.

DHA enhances gefitinib-induced inhibition of migration and invasion of NCI-H1975 cells. Representative results of the migration and invasion assays are presented in Fig. 4A. The migratory capabilities of NCI-H1975 cells were significantly reduced following treatment with gefitinib, and the addition of DHA potentiated the inhibitory actions of gefitinib (Fig. 4B). The effects of DHA co-administration were further investigated on the invasive capabilities of gefitinib-treated cancer cells. The number of NCI-H1975 cells that invaded the lower chamber was significantly reduced following treatment with a combination of DHA and gefitinib compared with treatment with gefitinib alone (Fig. 4C). These results demonstrated that DHA significantly enhanced the inhibitory effects of gefitinib on cancer cell migration and invasion.

DHA enhances gefitinib-induced downregulation of $p$-Akt, p-mTOR and p-STAT3 in NCI-H1975 cells. Western blot analysis revealed that $\mathrm{p}$-Akt protein expression levels were significantly downregulated following DHA and gefitinib co-administration. The $\beta$-actin was recognized as internal control; However, the levels of total Akt remained unaltered (Fig. 5A and B). In addition, DHA significantly enhanced the gefitinib-induced downregulation of $\mathrm{p}$-mTOR, whereas total mTOR levels remained unaffected across treatment groups (Fig. 5C and D). Similarly, p- but not total STAT3 protein expression levels were significantly downregulated following combined treatment with DHA and gefitinib (Fig. 5E and F). These results suggested that DHA may enhance the inhibitory actions of gefitinib on cellular migration and invasion possibly through the regulation of $\mathrm{p}-\mathrm{AKT} / \mathrm{p}-\mathrm{mTOR} / \mathrm{p}-\mathrm{STAT} 3$ pathways.

DHA enhances gefitinib-induced upregulation of Bax and downregulation of Bcl-2 in NCI-H1975 cells. Western blot 
A

B
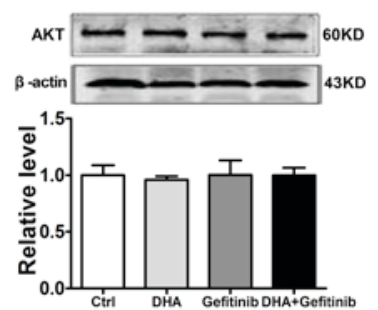

C
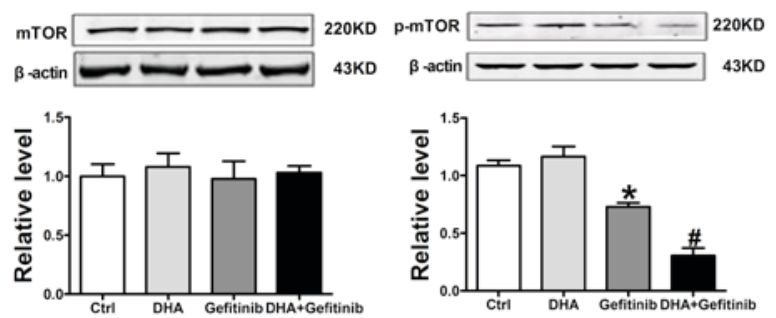

E
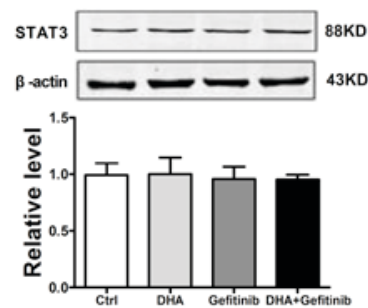

F

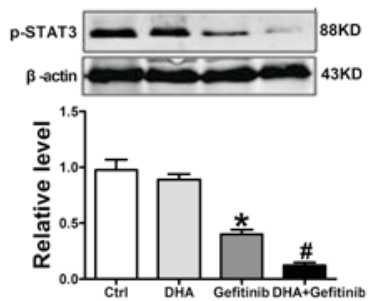

Figure 5. DHA enhanced the gefitinib-induced downregulation of p-Akt, p-mTOR and p-STAT3 in NCI-H1975 cells. Protein expression levels of (A) total Akt and (B) p-Akt; (C) total mTOR and (D) p-mTOR; and (E) total STAT3 and (F) p-STAT3. Expression levels of total proteins remained unaltered across treatment groups. Expression levels of phosphorylated proteins were significantly reduced following treatment with gefitinib. The effects of gefitinib were significantly enhanced following DHA co-administration. Cells in the Ctrl group received no treatment. Data are expressed as the mean \pm standard error of the mean of 3 independent experiments. ${ }^{*} \mathrm{P}<0.05$, ${ }^{* * *} \mathrm{P}<0.01$ vs. the $\mathrm{Ctrl}$ group; ${ }^{\#} \mathrm{P}<0.05,{ }^{\# \#} \mathrm{P}<0.01$ vs. the gefitinib group. DHA dihydroartemisinin; mTOR, mechanistic target of rapamycin; STAT, signa transducer and activator of transcription; $\mathrm{p}$, phosphorylated; Ctrl, control.

analysis revealed that Bax protein expression levels were significantly upregulated following treatment with gefitinib. Notably, Bax protein levels were significantly increased following DHA co-administration compared with cells treated with gefitinib alone (Fig. 6A). Conversely, Bcl-2 protein expression levels were significantly downregulated following treatment with gefitinib. Treatment with DHA and Gefitinib combined did not produce any further significant reduction in Bcl-2 levels, as presented in Fig. 6B.

\section{Discussion}

NSCLC is among the most common types of cancer and one of the leading causes of cancer-associated mortality. Drug resistance during cancer chemotherapy is a major concern in cancer therapeutics (22). Therefore, strategies aimed at increasing the efficacy of chemotherapy may help improve disease prognosis and improve the patients' quality of life.

A
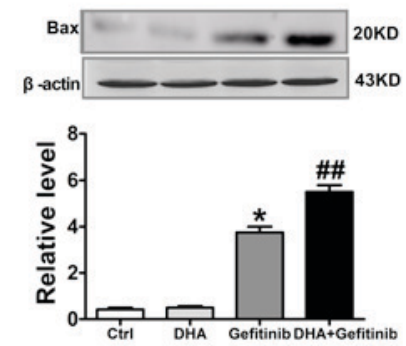

B
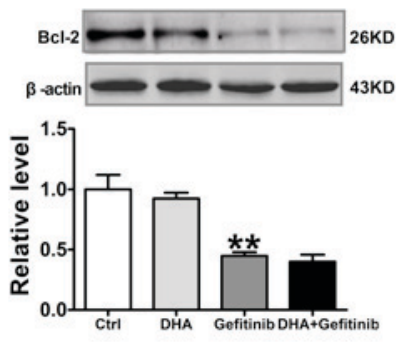

Figure 6. Effects of DHA and gefitinib co-administration on Bax and Bcl-2 protein expression levels in NCI-H1975 cells. (A) Treatment of NCI-H1975 cells with gefitinib significantly upregulated Bax protein expression levels. The effects of gefitinib were significantly enhanced following DHA co-administration. (B) Treatment of NCI-H1975 cells with gefitinib significantly downregulated $\mathrm{Bcl}-2$ protein expression levels. Cells in the Ctrl group received no treatment. Data are expressed as the mean \pm standard error of the mean of 3 independent experiments. ${ }^{*} \mathrm{P}<0.05,{ }^{* *} \mathrm{P}<0.01$ vs. the $\mathrm{Ctrl}$ group; ${ }^{\# \#} \mathrm{P}<0.01$ vs. the gefitinib group. DHA, dihydroartemisinin; Bcl-2, B-cell lymphoma-2; Bax, Bcl-2-associated X protein; Ctrl, control.

Natural products have garnered attention as a source for the development of novel anticancer drugs. Numerous natural products have exhibited anticancer potential and may prove useful, either as single agents or in combination with existing antineoplastic drugs, in the treatment of various types of cancer (23-26).

DHA is derived from artemisinin, a natural product isolated from Artemisia apiacea. It has previously been reported that DHA exhibited antitumor effects in several types of cancer (27). In accordance with previous studies, the present results demonstrated that DHA exhibited anticancer effects in NCI-H1975 cells. DHA appeared to suppress NCI-H1975 cellular proliferation in a concentration manner. A prominent role has previously been reported for mTOR in cancer. Activation of mTOR signaling pathways has been demonstrated to contribute to the initiation and progression of tumorigenesis $(28,29)$. Conversely, the inhibition of mTOR by rapamycin has been reported to enhance the chemosensitivity of cancer cells (30), and in NSCLC mTOR inhibition induced apoptosis and autophagy (31). In addition, it has been reported that DHA suppressed p-mTOR activity in ovarian cancer and rhabdomyosarcoma cells (32). Therefore, the present study evaluated the effects of DHA on mTOR. The result indicated that DHA inhibited the activity of p-mTOR. The western blot results revealed that administration of DHA alone had no effect on mTOR or p-mTOR, appearing only to potentiate the actions of gefitinib.

It has previously been demonstrated that DHA may enhance the efficacy of chemotherapy in the treatment of cancer (33). Therefore, the effects of the combination of DHA with the antineoplastic drug gefitinib were investigated in NCI-H1975 cells. Flow cytometric analysis demonstrated that the combination of DHA and gefitinib caused cell cycle arrest at the $\mathrm{G}_{0} / \mathrm{G}_{1}$ and $\mathrm{G}_{2} / \mathrm{M}$ phase. The percentage of cells in the G0/G1 phase was decreased by DHA+gefitinib. which was accompanied by a downregulation in Cdk1 and cyclin B1 protein expression levels. It has previously been demonstrated that Bcl-2 is a type of apoptosis-suppressing gene, which promotes cell survival by inhibiting adapters 
needed for activation of the proteases (caspases) (34). Bcl-2 elevation may enhance various types of cell survival and promote cancer progression and this activity has been observed in colon, prostate and lung cancers (35-37). Bax encodes a dominant-inhibitor of the Bcl-2 protein (38) and its upregulation may promote cell apoptosis in multiple types of cancer (38-42). Furthermore, it was revealed to induce apoptosis in NCI-H1975 cells, as well as a decrease in Bcl-2 and increase in Bax protein expression levels. The expression ratio of Bcl-2/Bax was also decreased (Fig. 6A and B). Notably, although gefitinib induced cell cycle arrest and apoptosis, in combination with DHA its effects were significantly potentiated. These results suggested that DHA may act synergistically with gefitinib in cancer cells.

To explore the molecular mechanisms underlying the anticancer effects of combined treatment with DHA and gefitinib, the protein levels of Akt, mTOR and STAT3 were evaluated. The Akt/mTOR/STAT3 signaling pathway is critical in the regulation of cancer cell growth, migration and apoptosis $(43,44)$. The present results revealed that $\mathrm{p}-\mathrm{Akt}, \mathrm{p}$-mTOR and p-STAT3 were significantly downregulated in NCI-H1975 cells that received a combination of DHA and gefitinib; however, total protein levels of Akt, mTOR and STAT3 remained unaltered across treatment groups. These results suggested that the synergistic effects of DHA and gefitinib may be exerted through the Akt/mTOR/STAT3 pathway. However, further experiments are required to elucidate the complex mechanisms underlying the anticancer effects of DHA and gefitinib.

In conclusion, the present study demonstrated that DHA enhanced the inhibitory effects of gefitinib on cancer cell proliferation. The combination of DHA with gefitinib significantly inhibited the growth and promoted the apoptosis of NCI-H1975 NSCLC cells. The mechanisms underlying the anticancer effects presently observed may involve regulation of the Akt/mTOR/STAT3 signaling pathway.

\section{References}

1. Jemal A, Bray F, Center MM, Ferlay J, Ward E and Forman D: Global cancer statistics. CA Cancer J Clin 61: 69-90, 2011

2. Song L, Xiong H, Li J, Liao W, Wang L, Wu J and Li M Sphingosine kinase-1 enhances resistance to apoptosis through activation of $\mathrm{PI} 3 \mathrm{~K} / \mathrm{Akt} / \mathrm{NF}-\kappa \mathrm{B}$ pathway in human non-small cell lung cancer. Clin Cancer Res 17: 1839-1849, 2011.

3. Chemotherapy in non-small cell lung cancer: A meta-analysis using updated data on individual patients from 52 randomised clinical trials. Non-small Cell Lung Cancer Collaborative Group. BMJ 311: 899-909, 1995.

4. Iwamoto Y, Mitsudomi T, Sakai K, Yamanaka T, Yoshioka H, Takahama M, Yoshimura M, Yoshino I, Takeda M and Sugawara S, et al: Randomized phase II study of adjuvant chemotherapy with long-term S-1 versus cisplatin $+\mathrm{S}-1$ in completely resected stage II-IIIA non-small cell lung cancer. Clin Cancer Res 21: 5245-5252, 2015.

5. Scagliotti GV, Fossati R, Torri V, Crinò L, Giaccone G, Silvano G, Martelli M, Clerici M, Cognetti F and Tonato M; Adjuvant Lung Project Italy/European Organisation for Research Treatment of Cancer-Lung Cancer Cooperative Group Investigators: Randomized study of adjuvant chemotherapy for completely resected stage I, II, or IIIA non-small-cell lung cancer. J Natl Cancer Inst 95: 1453-1461, 2003.

6. Cohen MH, Williams GA, Sridhara R, Chen G, McGuinn WD Jr, Morse D, Abraham S, Rahman A, Liang C, Lostritto R, et al: United States food and drug administration drug approval summary: Gefitinib (ZD1839; Iressa) tablets. Clin Cancer Res 10 1212-1218, 2004
7. Lynch TJ, Bell DW, Sordella R, Gurubhagavatula S, Okimoto RA, Brannigan BW, Harris PL, Haserlat SM, Supko JG, Haluska FG, et al: Activating mutations in the epidermal growth factor receptor underlying responsiveness of non-small-cell lung cancer to gefitinib. N Engl J Med 350: 2129-2139, 2004.

8. Hu CJ, Zhou L and Cai Y: Dihydroartemisinin induces apoptosis of cervical cancer cells via upregulation of RKIP and downregulation of bcl-2. Cancer Biol Ther 15: 279-288, 2014.

9. Dong Q, Chen L, Lu Q, Sharma S, Li L, Morimoto S and Wang G: Quercetin attenuates doxorubicin cardiotoxicity by modulating Bmi-1 expression. Br J Pharmacol 171: 4440-4454, 2014.

10. Zhang S, Ma Y, Jiang J, Dai Z, Gao X, Yin X, Xi W1 and Min W: Inhibition of urokinase-type plasminogen activator expression by dihydroartemisinin in breast cancer cells. Oncol Lett 7: 1375-1380, 2014.

11. Zhou HJ, Zhang JL, Li A, Wang Z and Lou XE: Dihydroartemisinin improves the efficiency of chemotherapeutics in lung carcinomas in vivo and inhibits murine Lewis lung carcinoma cell line growth in vitro. Cancer Chemother Pharmacol 66: 21-29, 2010.

12. Mi YJ, Geng GJ, Zou ZZ, Gao J, Luo XY, Liu Y2, Li N, Li CL, Chen YQ, Yu XY2 and Jiang J: Dihydroartemisinin inhibits glucose uptake and cooperates with glycolysis inhibitor to induce apoptosis in non-small cell lung carcinoma cells. PLoS One 10: e0120426, 2015.

13. Hermeking H: The miR-34 family in cancer and apoptosis. Cell Death Differ 17: 193-199, 2010.

14. Wang J, Wei H, Zhao B, Li M, Lv W, Lv L, Song B and Lv S: The reverse effect of X-ray irradiation on acquired gefitinib resistance in non-small cell lung cancer cell line NCI-H1975 in vitro. J Mol Histol 45: 641-652, 2014.

15. Fan Y, Chen M, Meng J, Yu L, Tu Y, Wan L, Fang K and Zhu W: Arsenic trioxide and resveratrol show synergistic anti-leukemia activity and neutralized cardiotoxicity. PLoS One 9: e105890, 2014.

16. Zhao X, Zhong H, Wang R, Liu D, Waxman S, Zhao L and Jing Y: Dihydroartemisinin and its derivative induce apoptosis in acute myeloid leukemia through Noxa-mediated pathway requiring iron and endoperoxide moiety. Oncotarget 6: 5582-5596, 2015.

17. Zhao C, Gao W and Chen T: Synergistic induction of apoptosis in A549 cells by dihydroartemisinin and gemcitabine. Apoptosis 19: 668-681, 2014

18. Maemondo M, Inoue A, Kobayashi K, Sugawara S, Oizumi S, Isobe $\mathrm{H}$, Gemma A, Harada M, Yoshizawa $\mathrm{H}$ and Kinoshita I: Gefitinib or chemotherapy for non-small-cell lung cancer with mutated EGFR. N Engl J Med 362: 2380-2388, 2010.

19. Yusuf SW, Kim P and Durand JB: Erlotinib or gefitinib for non-small-cell lung cancer. N Engl J Med 364: 2367-2368, 2011.

20. Matsui TA, Murata H, Sakabe T, Sowa Y, Horie N, Nakanishi R, Sakai T and Kubo T: Sulforaphane induces cell cycle arrest and apoptosis in murine osteosarcoma cells in vitro and inhibits tumor growth in vivo. Oncol Rep 18: 1263-1268, 2007.

21. Jakubikova J, Bao Y and Sedlak J: Isothiocyanates induce cell cycle arrest, apoptosis and mitochondrial potential depolarization in HL-60 and multidrug-resistant cell lines. Anticancer Res 25: 3375-3386, 2005

22. Chang A: Chemotherapy, chemoresistance and the changing treatment landscape for NSCLC. Lung Cancer 71: 3-10, 2011.

23. Simmons TL, Andrianasolo E, McPhail K, Flatt $\mathrm{P}$ and Gerwick WH: Marine natural products as anticancer drugs. Mol Cancer Ther 4: 333-342, 2005.

24. Gordaliza M: Natural products as leads to anticancer drugs. Clinical Translational Oncol 9: 767-776, 2007.

25. Altmann KH and Gertsch J: Anticancer drugs from nature-natural products as a unique source of new microtubule-stabilizing agents. Nat Prod Rep 24: 327-357, 2007.

26. Newman DJ and Cragg GM: Natural products as sources of new drugs over the last 25 years. J Nat Prod 70: 461-477, 2007.

27. Sun H, Meng X, Han J, Zhang Z, Wang B, Bai X and Zhang X: Anti-cancer activity of DHA on gastric cancer-an in vitro and in vivo study. Tumour Biol 34: 3791-3800, 2013.

28. Follo MY, Manzoli L, Poli A, McCubrey JA and Cocco L: PLC and PI3K/Akt/mTOR signalling in disease and cancer. Adv Biol Regul 57: 10-16, 2015.

29. Cornu M, Albert V and Hall MN: $m T O R$ in aging, metabolism, and cancer. Curr Opin Genet Dev 23: 53-62, 2013.

30. Faried LS, Faried A, Kanuma T, Nakazato T, Tamura T, Kuwano $\mathrm{H}$ and Minegishi T: Inhibition of the mammalian target of rapamycin (mTOR) by rapamycin increases chemosensitivity of CaSki cells to paclitaxel. Eur J Cancer 42: 934-947, 2006. 
31. Li YC, He SM,He ZX, Li M, Yang Y, Pang JX, Zhang X, Chow K Zhou Q, Duan W, et al: Plumbagin induces apoptotic and autophagic cell death through inhibition of the PI3K/Akt $/ \mathrm{mTOR}$ pathway in human non-small cell lung cancer cells. Cancer Lett 344: 239-259, 2014.

32. Feng X, Li L, Jiang H, Jiang K, Jin Y and Zheng J: Dihydroartemisinin potentiates the anticancer effect of cisplatin via mTOR inhibition in cisplatin-resistant ovarian cancer cells: involvement of apoptosis and autophagy. Biochem Biophys Res Commun 444: 376-381, 2014.

33. Wu GS, Lu JJ, Guo JJ, Huang MQ, Gan L, Chen XP and Wang YT: Synergistic anti-cancer activity of the combination of dihydroartemisinin and doxorubicin in breast cancer cells Pharmacol Rep 65: 453-459, 2013.

34. Adams JM and Cory S: The Bcl-2 protein family: Arbiters of cell survival. Science 281: 1322-1326, 1998

35. Sheng H, Shao J, Morrow JD, Beauchamp RD and DuBois RN Modulation of apoptosis and $\mathrm{Bcl}-2$ expression by prostaglandin E2 in human colon cancer cells. Cancer Res 58: 362-366, 1998.

36. McDonnell TJ, Troncoso P, Brisbay SM, Logothetis C, Chung LW, Hsieh JT, Tu SM and Campbell ML: Expression of the protooncogene bcl-2 in the prostate and its association with emergence of androgen-independent prostate cancer. Cancer Res 52: 6940-6944, 1992.

37. Katz HR: bcl-2 protein in non-small-cell lung carcinoma. N Engl J Med 330: 221, 1994
38. Miyashita T, Krajewski S, Krajewska M, Wang HG, Lin HK, Liebermann DA, Hoffman B and Reed JC: Tumor suppressor p53 is a regulator of bcl-2 and bax gene expression in vitro and in vivo. Oncogene 9: 1799-1805, 1994.

39. Zhang L, Yu J, Park BH, Kinzler KW and Vogelstein B: Role of BAX in the apoptotic response to anticancer agents. Science 290: 989-992, 2000

40. Choudhuri T, Pal S, Agwarwal ML, Das T and Sa G: Curcumin induces apoptosis in human breast cancer cells through p53-dependent Bax induction. FEBS Lett 512: 334-340, 2002.

41. Brambilla E, Negoescu A, Gazzeri S, Lantuejoul S, Moro D, Brambilla C and Coll JL: Apoptosis-related factors p53, Bcl2, and Bax in neuroendocrine lung tumors. Am J Pathol 149: 1941-1952, 1996.

42. Gupta S, Afaq F and Mukhtar H: Involvement of nuclear factor-kappa B, Bax and Bcl-2 in induction of cell cycle arrest and apoptosis by apigenin in human prostate carcinoma cells. Oncogene 21: 3727-3738, 2002.

43. Sakamoto KM, Grant S, Saleiro D, Crispino JD, Hijiya N, Giles F, Platanias L and Eklund EA: Targeting novel signaling pathways for resistant acute myeloid leukemia. Mol Genet Metab 114: 397-402, 2015

44. He SQ, Gao M, Fu YF and Zhang YN: Glycyrrhizic acid inhibits leukemia cell growth and migration via blocking AKT/mTOR/STAT3 signaling. Int J Clin Exp Pathol 8: 5175-5181, 2015. 